\title{
Magnetic resonance imaging of pulmonary arterial compliance after pulmonary endarterectomy
}

\author{
To the Editor:
}

Pulmonary endarterectomy (PEA) is the treatment of choice of chronic thromboembolic pulmonary hypertension (CTEPH) [1]. However, successfully operated patients may continue to suffer from dyspnoea and limitation of exercise capacity, despite improvement or even normalisation of pulmonary artery pressure (PAP), cardiac output (CO) and pulmonary vascular resistance (PVR) [2]. This absence of complete symptomatic recovery has been explained by a decreased right ventricular (RV) function reserve due to persistent increased afterload $[3,4]$, related to decreased pulmonary arterial compliance (PCa) more than to mildly increased PVR $[5,6]$. There is therefore interest in assessing PCa in patients during the follow-up of PEA.

Estimation of PCa commonly relies on the invasively measured ratio of stroke volume (SV) and pulse pressure (PP). This approach is limited by the fact that pulmonary circulation is not a closed system and blood leaves through the pulmonary resistive vessels, therefore SV/PP ratio over-estimates $\mathrm{PCa}$ considerably [7]. This problem can be addressed by the pulse pressure method (PPM), which calculates PCa from the instantaneous pulmonary artery (PA) flow and a two-element windkessel model with an initial value of SV/PP and PVR [8]. Another approach is to estimate PCa using systolic and diastolic cross sectional area or flow imaging of SV by cardiac magnetic resonance (CMR) in combination or not with the right heart catheterisation (RHC) assessment of PP $[9,10]$.

We wondered whether CMR imaging of the PA could be a clinically useful addition to RHC determination of PCa for the evaluation of CTEPH patients in the follow-up of successful PEA. We also compared patients with proximal versus distal lesions to better understand the role of anatomical location of the disease in the assessment of PCa.

The present study was a retrospective evaluation of a consecutive cohort of patients who underwent PEA at our institution (Fondazione IRCCS Policlinico San Matteo, Pavia, Italy) and in whom CMR, RHC and a Bruce test were successively performed during the same day, before PEA and 12 months after surgery. All patients signed an informed consent agreement, approved by the institutional review board of Fondazione IRCCS Policlinico San Matteo (Pavia, Italy) for longitudinal, nonpharmacological, non-sponsored studies, which complies with the Italian legislation (Codex on Privacy, D. Lgs. 30 giugno 2003, n. 196).

The anaesthesiological and surgical methods used have been reported previously [11]. RHC was performed as recommended in the guidelines of the European Respiratory Society and European Society of Cardiology [12]. The response variable was exercise capacity assessed using the modified Bruce protocol and expressed in distance achieved in metres. CMR was performed using a $1.5 \mathrm{~T}$ scanner (Siemens Synphony, Erlangen, Germany) with a phased array cardiac coil and ECG gating.

RHC PCa was calculated as (SV/PP divided by 1.76)+0.1, in conformance to the PPM model [8]. CMR-derived estimates included: relative cross-sectional relative area change (RAC) of the PA (RAC= $((\max A-\min A) / \min A) \times 100)$, area compliance calculated as absolute change in lumen area for a given change in pressure $((\max \mathrm{A}-\mathrm{min} \mathrm{A}) / \mathrm{PP})$, area distensibility calculated as the ratio of RAC and $\mathrm{PP}$, vessel

@ERSpublications

In CTEPH patients who have undergone pulmonary endarterectomy, exercise capacity is better associated with changes in invasively measured pulmonary arterial compliance than with changes in magnetic resonance imaging of pulmonary artery stiffness http://bit.ly/30DAWUj

Cite this article as: Ghio S, Crimi G, Guida S, et al. Magnetic resonance imaging of pulmonary arterial compliance after pulmonary endarterectomy. Eur Respir J 2020; 55: 1902171 [https://doi.org/10.1183/ 13993003.02171-2019]. 
wall stiffness $(\beta)$ calculated as (ln (PAPs/PAPd))/(2 RAC) and CMR compliance as the ratio of CMR flow imaging SV $\left(\mathrm{SV}_{\mathrm{CMR}}\right)$ and PP.

Data were described as mean $\pm \mathrm{SD}$ and compared between the groups (proximal-mid versus distal disease) with the t-test. Haemodynamic and CMR parameters before and after surgery, were compared within individual subjects, by fitting a mixed model for repeated measure, response was defined as total distance at Bruce test. Time (surgery) effect was explored between the groups (proximal versus distal CTEPH) by including the group and interaction term time $\times$ group in the model; other covariates were included into the model to assess independent predictive impact on response. Post hoc comparisons were adjusted by the Tukey honest significant difference correction; a two-sided probability value $\leqslant 0.05$ was considered significant. Data were analysed in $\mathrm{R}$ version 3.5.3.

The study included 86 patients, aged $61 \pm 13$ years, 46 with proximal and 41 with distal CTEPH, with a WHO functional class III or IV in 60 of them. PEA was associated with improvement in the Bruce test distance from $181 \pm 201$ to $544 \pm 286 \mathrm{~m}$.

At RHC, cardiac index improved after PEA from $2.3 \pm 0.7$ to $2.7 \pm 0.5 \mathrm{~L} \cdot \mathrm{min}^{-1} \cdot \mathrm{m}^{-2}$, mean PAP from $42 \pm 12$ to $19 \pm 6 \mathrm{mmHg}$, PVR from $776 \pm 378$ to $216 \pm 105$ dyne.s $\cdot \mathrm{cm}^{-5}$ and SV/PP from $1.2 \pm 0.7$ to $3.1 \pm 1.1 \mathrm{~mL} \cdot \mathrm{mmHg}^{-1}$ (all $\mathrm{p}<001)$. The time constant PVR $\times$ PCa remained unchanged in both proximal and distal CTEPH patients. At CMR, area compliance increased after PEA from $4.0 \pm 3.6$ to $6.8 \pm 4.4 \mathrm{~mm}^{2} \cdot \mathrm{mmHg}^{-1}, \mathrm{SV}_{\mathrm{CMR}} / \mathrm{PP}$ from $1.3 \pm 0.6$ to $3.2 \pm 1.2 \mathrm{~mL} \cdot \mathrm{mmHg}^{-1}$, RAC from $23 \pm 18$ to $25 \pm 17 \%$ and RAC/PP from $0.5 \pm 0.5$ to $1.2 \pm 0.91 \% \cdot \mathrm{mmHg}^{-1}$ while vessel wall stiffness decreased from $703 \pm 1832$ to $167 \pm 214$ arbitrary units (all $\mathrm{p}<0.001$, except for vessel wall stiffness $\mathrm{p}=0.18$ and RAC $\mathrm{p}=0.24$ ). All the RHC and CMR changes after PEA were similar and not significantly different in patients with proximal or distal lesions. This is illustrated by PEA-related changes in PCa and Bruce test in figure 1.

a)
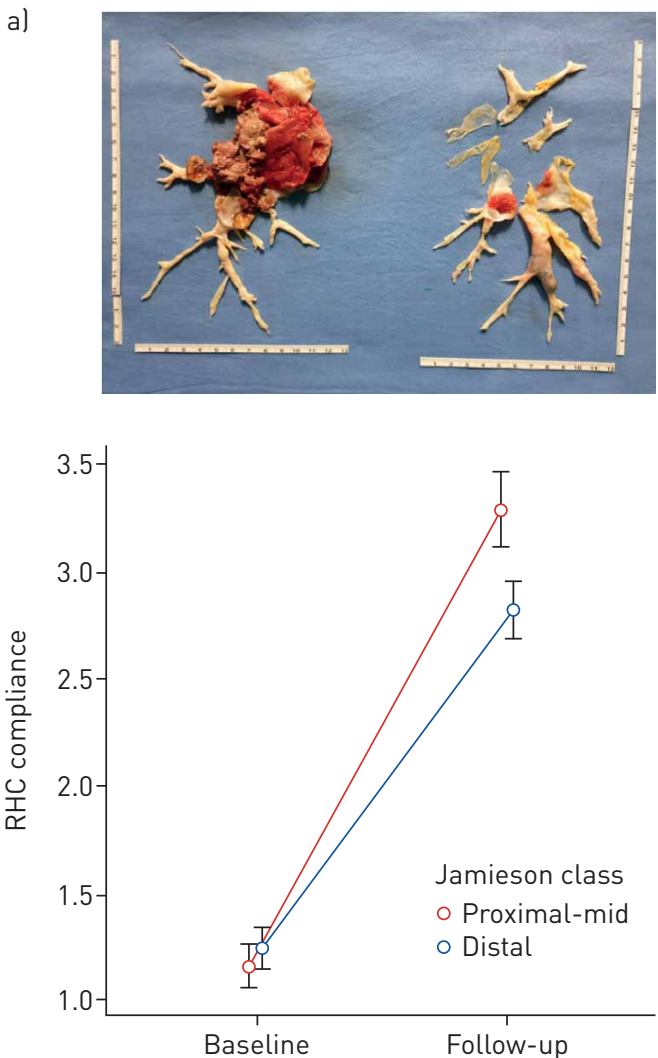

b)
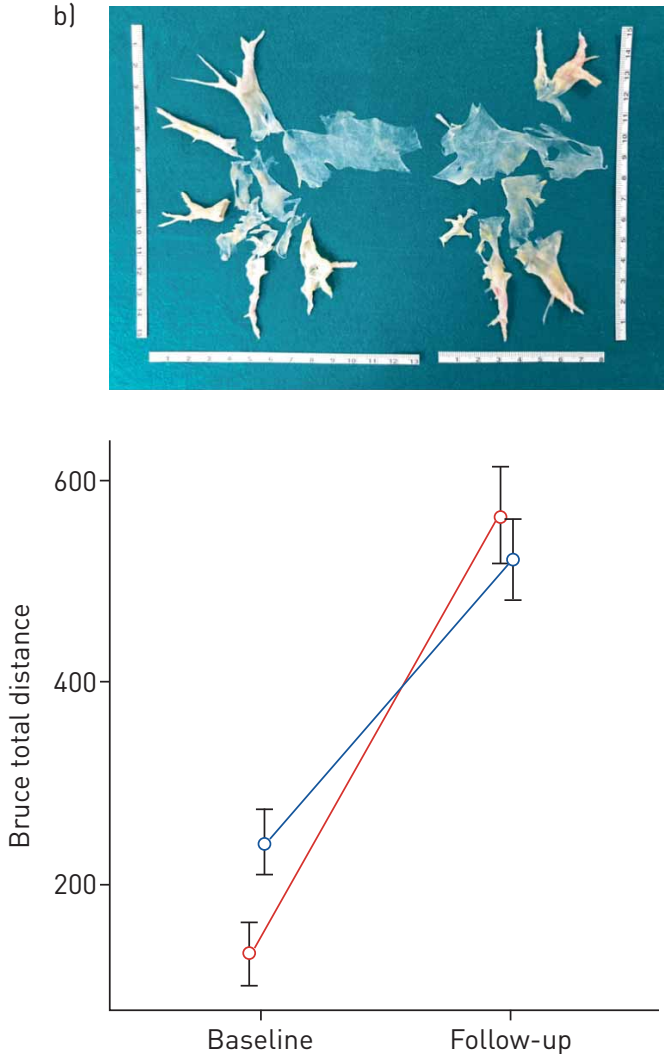

FIGURE 1 Changes in a) pulmonary arterial compliance calculated at right heart catheterisation (RHC) and b) metres walked at Bruce test from baseline to post surgery in patients with Jamieson type 1-2 disease (red line and circles) and in patients with Jamieson type 3 disease (blue line and circles). Post pulmonary endarterectomy Jamieson type 1-2 versus distal Jamieson type 3 casts are illustrated in the upper part of the figure. Data were analysed in a mixed multivariable model for repeated measure, class effect, time (surgery) effect and the interaction between class and time were included as covariates. Statistical significance as follows. a) Jamieson class effect $p=0.193$; time effect $p<0.001$; Jamieson class $\times$ time $p=0.018$. b) Jamieson class effect $p=0.710$; time effect $p<0.001$; Jamieson class $\times$ time $p=0.050$. 
PCa was the only independent haemodynamic parameter associated with improvement in exercise capacity by Bruce exercise test before/after PEA in a multivariable model $(\mathrm{p}=0.018)$, while mean PAP and PVR were nonsignificant predictors ( $\mathrm{p}=0.692$ and 0.095 , respectively). When CMR-based indices of PA structure and function were incorporated in a multivariable model, only $\mathrm{SV}_{\mathrm{CMR}} / \mathrm{PP}$ independently predicted exercise capacity but with borderline significance $(p=0.051)$. However, RHC-derived assessment of PCa but not $\mathrm{SV}_{\mathrm{CMR}} / \mathrm{PP}$ was independently associated with improvement in exercise capacity when both were included in a bivariable model $(\mathrm{p}<0.001$ and $\mathrm{p}=0.588$, respectively).

The observation that the anatomical location of vascular obstruction and relief by PEA surgery did not affect the time constant of the pulmonary circulation is in keeping with previous demonstration [13]. RV afterload is determined by a dynamic interplay between pulmonary vascular resistance, compliance and wave reflection [14]. Therefore the present results suggest a negligible contribution of wave reflection as PCa is tightly related to PVR and, thus, RV hydraulic load is essentially predicted by PCa and PVR only, with a hyperbolic relationship so that PCa predominates in the presence of normal or only mildly increased PAP [15]. On the other hand, CMR imaging of PA stiffness is necessarily limited to the proximal part of the pulmonary arterial tree, so that proximal PA compliance contributes to no more than $20 \%$ of the total compliance of the pulmonary vascular bed [13]. This explains why CMR imaging of PA stiffness did not predict exercise capacity in the present study.

In conclusion, the present results show that CMR imaging of PA dimensions cannot replace RHC and is of no added value to RHC for the determination of PCa before and after successful PEA in CTEPH patients with either proximal or distal thrombotic lesions.

Stefano Ghio ${ }^{1}$, Gabriele Crimi $\odot^{1}$, Stefania Guida $\odot^{1}$, Adele Valentini ${ }^{2}$, Anna Celentano ${ }^{3}$, Maurizio Pin $^{3}$, Claudia Raineri ${ }^{1}$, Annalisa Turco ${ }^{1}$, Laura Scelsi ${ }^{1}$, Luigi Oltrona Visconti ${ }^{1}$, Robert Naeije $^{4}$ and Andrea Maria D'Armini ${ }^{3,5}$

${ }^{1}$ Division of Cardiology, Fondazione IRCCS Policlinico San Matteo, Pavia, Italy. ${ }^{2}$ Institute of Radiology, Fondazione IRCCS Policlinico San Matteo, Pavia, Italy. ${ }^{3}$ Division of Cardiac Surgery, Fondazione IRCCS Policlinico San Matteo, Pavia, Italy. ${ }^{4}$ Free University of Brussels, Brussels, Belgium. ${ }^{5}$ Dept of Surgical, Clinical, Diagnostic and Pediatric Sciences, University of Pavia School of Medicine, Pavia, Italy.

Correspondence: Stefano Ghio, Divisione di Cardiologia, Policlinico S. Matteo, Piazza Golgi 1, 27100 Pavia, Italy. E-mail: s.ghio@smatteo.pv.it

Received: 08 Nov 2019 | Accepted after revision: 12 Jan 2020

Author contributions: S. Ghio made substantial contributions to conception and to the design of the study and to interpretation of data; drafted the submitted article; and provided final approval of the version to be published. S. Ghio takes responsibility for (is the guarantor of) the content of the manuscript, including the data and analysis. G. Crimi, S. Guida, A. Valentini, A. Celentano, M. Pin, C. Raineri, A. Turco, L. Oltrona Visconti, R. Naeije and A.M. D'Armini have made substantial contributions to conception of the study, to interpretation of data; have revised the manuscript critically for important intellectual content and has provided final approval of the version to be published; G. Crimi has also analysed the data.

Conflict of interest: None declared.

\section{References}

1 Kim NH, Delcroix M, Jais X, et al. Chronic thromboembolic pulmonary hypertension. Eur Respir J 2019; 53: 1801915.

2 Lang IM M. Update on chronic thromboembolic pulmonary hypertension. Circulation 2014; 130: 508-518.

3 Bonderman D, Martischnig AM, Vonbank K, et al. Right ventricular load at exercise is a cause of persistent exercise limitation in patients with normal resting pulmonary vascular resistance after pulmonary endarterectomy. Chest 2011; 139: 122-127.

4 Claessen G, La Gerche A, Dymarkowski S, et al. Pulmonary vascular and right ventricular reserve in patients with normalized resting haemodynamics after pulmonary endarterectomy. J Am Heart Assoc 2015; 4: e001602.

5 Ghio S, Morsolini M, Corsico A, et al. Pulmonary arterial compliance and exercise capacity after pulmonary endarterectomy. Eur Respir J 2014; 43: 1403-1409.

6 Corsico AG, D’Armini AM, Conio V, et al. Persistent exercise limitation after successful pulmonary endoarterectomy: frequency and determinants. Respir Res 2019; 20: 34

7 Stergiopulos N, Meister JJ, Westerhof N. Evaluation of methods for estimation of total arterial compliance. Am J Physiol 1995; 268: H1540-H1548.

8 Segers $\mathrm{P}$, Brimioulle S, Stergiopulos N, et al. Pulmonary arterial compliance in dogs and pigs: the three-element windkessel model revisited. Am J Physiol 1999; 277: H725-H723.

9 Gan CT, Lankhaar JW, Westerhof N, et al. Noninvasively assessed pulmonary artery stiffness predicts mortality in pulmonary arterial hypertension. Chest 2007; 132: 1906-1912.

10 Stevens GR, Garcia-Alvarez A, Sahni S, et al. RV dysfunction in pulmonary hypertension is independently related to pulmonary artery stiffness. JACC Cardiovasc Imaging 2012; 5: 378-387.

11 Morsolini M, Nicolardi S, Milanesi E, et al. Evolving surgical techniques for pulmonary endarterectomy according to the changing features of chronic thromboembolic pulmonary hypertension patients during 17 -year single center experience. J Thorac Cardiovasc Surg 2012; 144: 100-107. 
12 Galiè N, Humbert M, Vachiery JL, et al. 2015 ESC/ERS Guidelines for the diagnosis and treatment of pulmonary hypertension: The Joint Task Force for the Diagnosis and Treatment of Pulmonary Hypertension of the European Society of Cardiology (ESC) and the European Respiratory Society (ERS). Eur Respir J 2015; 46: 903-975.

13 Saouti N, Westerhof N, Helderman F, et al. RC time constant of single lung equals that of both lungs together: a study in chronic thromboembolic pulmonary hypertension. Am J Physiol Heart Circ Physiol 2009; 297: $\mathrm{H} 2154-\mathrm{H} 2160$.

14 Sniderman AD, Fitchett DH. Vasodilators and pulmonary arterial hypertension: the paradox of therapeutic success and clinical failure. Int J Cardiol 1988; 20: 173-181.

15 Saouti N, Westerhof N, Helderman F, et al. Right ventricular oscillatory power is a constant fraction of total power irrespective of pulmonary artery pressure. Am J Respir Crit Care Med 2010; 182: 1315-1320.

Copyright (C)ERS 2020 